\title{
BOUND-STATE SOLUTIONS OF THE MODIFIED KLEIN-GORDON AND SCHRÖDINGER EQUATIONS FOR ARBITRARY L-STATE WITH THE MODIFIED MORSE POTENTIAL IN THE SYMMETRIES OF NONCOMMUTATIVE QUANTUM MECHANICS
}

\author{
Abdelmadjid Maireche ${ }^{(D)}$ \\ Laboratory of Physics and Material Chemistry, Physics department, \\ Sciences Faculty, University of M'sila, \\ BP 239 Chebilia-M'sila, Algeria \\ e-mail: abdelmadjid.maireche@univ-msila.dz
}

(Received 08 May 2020; in final form 27 November 2020; accepted 03 December 2020; published online 23 February 2021)

\begin{abstract}
In this work, approximate analytical solutions of both modified Klein-Gordon equation and Schrödinger equation in noncommutative relativistic and nonrelativistic three-dimensional real space have been explored by using the Pekeris approximation scheme to deal with the centrifugal term, Bopp's shift method and standard perturbation theory. We present the bound-state energy equation with a newly proposed potential called the modified Morse potential under the condition of equal scalar and vector potentials. The potential is a superposition of the Morse potential and some exponential radial terms. The aim of combining these potentials is to have an extensive application. We show that the new energy depends on the global parameters $\left(\Theta^{c}\right.$ and $\left.\sigma^{c}\right)$ characterizing the noncommutativity space-space and the potential parameter $\left(D_{e}, r_{e}, \alpha\right)$ in addition to the Gamma function and the discreet atomic quantum numbers $(j, l, s, m)$. The present results are applied in calculating both the energy spectrum for a few heterogeneous $(\mathrm{LiH}, \mathrm{HCl}, \mathrm{NO})$ and homogeneous $\left(\mathrm{H}_{2}, \mathrm{I}_{2}, \mathrm{O}_{2}\right)$ diatomic molecules. We have also discussed some special cases of physical importance.

Key words: Klein-Gordon equation, Schrödinger equation, the Morse potential, noncommutative quantum mechanics, star products.
\end{abstract}

DOI: https://doi.org/10.30970/jps.25.1002

\section{INTRODUCTION}

It is a well-known fact that when a single particle or diatomic molecules are in a strong potential field, relativistic effect must be considered, leadi$\mathrm{ng}$ to the relativistic quantum mechanical description of studied systems. This has been interesting in the analytical and approximation solutions of the three fundamental equations, Schrödinger; equation, KleinGordon equation and Dirac equation in the field of chemistry, physics and pure and applied mathematics. In recent years, some authors have studied some typical diatomic molecule empirical potentials such as the DengFan potential [1], the Pöschl-Teller potential [2]. In 2004, L. Z. Yi et al. [3] studied the Klein-Gordon equation with the Rosen-Morse-type potentials and obtained the energies and corresponding $s$-state wave solutions using hypergeometric functions. The Morse potential is widely used both in physics and in chemistry [4]. In 2005, the Morse potential has been studied for any $l$ states with hypergeometric functions analytically and numerically as well as by using the Pekeris approximation and the Nikiforov-Uvarov method [5]. In 2007, C. E. Burkhardt et al. studied vibration-rotation coupling in a Morse oscillator [6]. In 2010, O. Bayrak et al. [7] studied the relativistic spin-0 particles under the rotating Morse oscillator. In 2011, S. M. Ikhdair [8] obtained an approximate $k$ state solution of the Dirac equation for the generalized Morse potential under spin and pseudospin symmetry. In 2013, Chun-Sheng Jia et al. studied spinless energies of the Morse potential energy model [9]. In 2015, Xiang-Jun
Xie et al. obtained solutions of the Klein-Gordon equation with the Morse potential energy model in higher spatial dimensions [10]. In 2016, P. Zhang et al. [11] obtained solutions of the Dirac equation with the Morse potential energy model in higher spatial dimensions. This potential dates back to the third decade of the last century; in 1929, Morse [12] proposed a three-parameter potential energy function for the diatomic such as $\left(\mathrm{H}_{2}, \mathrm{HCl}, \mathrm{I}_{2}\right.$, $\mathrm{CO}, \mathrm{LiH})$ and even polyatomic molecules. The solutions of the Schrödinger equation with Morse potential for the states have been found exactly in [12-14]. For the case $l \neq 0$, the wave equation can only be solved by using perturbation and approximation. In particular, the Morse potential will reduce to the harmonic oscillator in the harmonic limit, it is ideal, and a typical anharmonic potential permits an exact mathematical treatment [15]. In 2019, P. O. Okoi et al. studied the Hellmann-generalized Morse potential and obtained the bound state energy eigenvalues for $\mathrm{N}_{2}, \mathrm{CO}, \mathrm{NO}, \mathrm{CH}$ and $\mathrm{HCl}$ diatomic molecules [16]. S. Miraboutalebi et al. [17] employed the Laplace transformation method to derive the exact solution of the $N$-dimensional Schrödinger equation with the Morse potential. In 2012, A. Arda et al. [18] employed the Laplace transformation method to derive the exact solution of the one dimensional Schrödinger equation with the Morse potential.

This work, motivated by many various recent studies such as the non-renormalizable electroweak interaction, quantum gravity, string theory, the noncommutative relativistic and nonrelativistic quantum mechanics has attracted much attention of physicists [19-26]. 
Noncommutativity in space-time is not a new or modern idea; it was proposed by W. Heisenberg in 1930 and then developed by H. Snyder in 1947. On the other hand, to the best of our knowledge, the Klein-Gordon equation has not been studied with the Morse potential, a study that may enable the extension of the interaction of spin- 0 particles. The main objective of this work is to develop the study by Purohit et al. within the framework of the Klein-Gordon equation and the Schrödinger equation but in the context of symmetries of noncommutative quantum mechanics, in order to investigate the microscopic scales and gain more scientific knowledge of elementary particles and diatomic molecular in the field of nano-scales. The relativistic and nonrelativistic energy levels under the modified Morse potential have not been obtained yet in the context of the noncommutative quantum mechanics NCQM. Furthermore, we hope to find new applications and profound physical interpretations using a new, updated model of the modified Morse potential. This new potential takes the form:

$$
\begin{gathered}
\left\{\begin{array}{c}
V_{\mathrm{mp}}(r)=a_{v}\left[1-\exp \left(-\alpha\left(r-r_{e}\right)\right)\right]^{2} \\
S_{\mathrm{mp}}(r)=a_{s}\left[1-\exp \left(-\alpha\left(r-r_{e}\right)\right)\right]^{2}
\end{array}\right. \\
\Rightarrow\left\{\begin{array}{c}
V_{\mathrm{mp}}(\hat{r})=V_{\mathrm{mp}}(r)-\frac{\partial V_{\mathrm{mp}}(r)}{\partial r} \frac{\mathbf{L} \Theta}{2 r} \\
+O\left(\Theta^{2}\right) \\
S_{\mathrm{mp}}(\hat{r})=S_{\mathrm{mp}}(r)-\frac{\partial S_{\mathrm{mp}}(r)}{\partial r} \frac{\mathbf{L} \Theta}{2 r} \\
+O\left(\Theta^{2}\right),
\end{array}\right.
\end{gathered}
$$

where $\vec{L} \vec{\Theta}=\mathbf{L} \Theta$, while $a_{v}$ and $a_{s}$ are two constant parameters, $r_{e}$ is the equilibrium bond length, $a_{v}=$ $D_{e}$ is the dissociation energy and $\alpha$ is the range of the potential, while $r$ is the interatomic or interparticle distance. The new structure of is NCQM based on new covariant noncommutative canonical commutations relations CNCCRs in the Schrödinger, Heisenberg and Interactions pictures (SP, HP and IP), respectively, as follows $[27-30]$ :

$$
\begin{aligned}
{\left[\hat{x}_{\mu}^{\mathrm{S}}{ }^{*} \hat{p}_{\nu}^{\mathrm{S}}\right] } & =\left[\hat{x}_{\mu}^{\mathrm{H}}(t) * \hat{p}_{\nu}^{\mathrm{H}}(t)\right]=\left[\hat{x}_{\mu}^{\mathrm{I}}(t),{ }^{\wedge} \hat{p}_{\nu}^{\mathrm{I}}(t)\right] \\
& =i \hbar_{\mathrm{eff}} \delta_{\mu \nu} \\
{\left[\hat{x}_{\mu}^{\mathrm{S}}, \wedge^{\mathrm{S}}\right] } & =\left[\hat{x}_{\nu}^{\mathrm{H}}(t) * \wedge^{\mathrm{H}}(t)\right]=\left[\hat{x}_{\mu}^{\mathrm{I}}(t) * \hat{x}_{\nu}^{\mathrm{I}}(t)\right] \\
& =i \theta_{\mu \nu}
\end{aligned}
$$

We have generalized the CNCCRs to include HP and IP. It should be noted that, in our calculation, we have used the natural units $c=\hbar=1$. Here $\hbar_{\mathrm{eff}} \simeq \hbar$ is the effective Plank constant, $\left(\theta^{\mu \nu}=\epsilon^{\mu \nu} \theta\right.$ is the non-commutative parameter), which is an infinitesimal parameter if it is compared to the energy values and elements of the antisymmetric $3 \times 3$ real matrix, and $\delta_{\mu \nu}$ is the identity matrix. The symbol denotes the WeylMoyal star product, which is generalized between two ordinary functions $(f g)(x)$ to the new modified form $(\hat{f} \hat{g})(\hat{x})=(f * g)(x)$ in the symmetries of (RNC: 3D$\mathrm{RS})$ as follows [31-38]:

$$
\begin{aligned}
(f * g)(x) & =\exp \left(i \epsilon^{\mu \nu} \theta \partial_{\mu}^{x} \partial_{\mu}^{x}\right)(f g)(x) \\
& \left.\approx(f g)(x)-\frac{i \epsilon^{\mu \nu} \theta}{2} \partial_{\mu}^{x} f \partial_{\mu}^{x} g\right\rfloor_{x^{\mu}=x^{\nu}}+O\left(\theta^{2}\right)
\end{aligned}
$$

The indices $(\mu, \nu=1,2,3)$ and $O\left(\theta^{2}\right)$ stand for the second and higher-order terms of the NC parameter. Physically, the term $\left.\left(-\frac{i \epsilon^{\mu \nu} \theta}{2} \partial_{\mu}^{x} f \partial_{\mu}^{x} g\right\rfloor_{x^{\mu}=x^{\nu}}\right)$ in Eq. (3) presents the effects of space-space noncommutativity properties. Furthermore, the two new unified operators $\hat{\zeta}_{\mu}^{\mathrm{H}}(t)=\left(\hat{x}_{\mu}^{\mathrm{H}} \vee \hat{p}_{\mu}^{\mathrm{H}}\right)(t)$ and $\hat{\zeta}_{\mu}^{\mathrm{I}}(t)=\left(\hat{x}_{\mu}^{\mathrm{I}} \vee \hat{p}_{\mu}^{\mathrm{I}}\right)(t)$ in HP and IP depend on the corresponding new operators $\zeta_{\mu}^{\mathrm{S}}(t)=\hat{x}_{\mu}^{\mathrm{S}} \vee \hat{p}_{\mu}^{\mathrm{S}}$ in SP from the following projections relations, respectively:

$$
\begin{aligned}
& \left\{\begin{array}{c}
\zeta_{\mu}^{\mathrm{H}}(t)=\exp \left(i \hat{H}_{\mathrm{rmp}} T\right) \zeta_{\mu}^{\mathrm{S}} \exp \left(-i \hat{H}_{\mathrm{rmp}} T\right) \\
\zeta_{\mu}^{\mathrm{I}}(t)=\exp \left(i \hat{H}_{\mathrm{omp}} T\right) \zeta_{\mu}^{\mathrm{S}}(t) \exp \left(-i \hat{H}_{\mathrm{omp}} T\right)
\end{array},\right. \\
& \begin{array}{c}
\Longrightarrow \\
\left\{\begin{array}{l}
\hat{\zeta}_{\mu}^{\mathrm{H}}(t)=\exp \left(i \hat{H}_{\mathrm{rnc}}^{\mathrm{mp}} T\right) * \hat{\zeta}_{\mu}^{\mathrm{S}} * \exp \left(-i \hat{H}_{\mathrm{rnc}}^{\mathrm{mp}} T\right) \\
\hat{\zeta}_{\mu}^{\mathrm{I}}(t)=\exp \left(i \hat{H}_{\text {onc }}^{\mathrm{mp}} T\right) * \hat{\zeta}_{\mu}^{\mathrm{S}} * \exp \left(-i \hat{H}_{\text {onc }}^{\mathrm{mp}} T\right)
\end{array}\right.
\end{array}
\end{aligned}
$$

Here $T=t-t_{0}, \zeta_{i}^{\mathrm{S}}=x_{i} \vee p_{i}, \zeta_{i}^{\mathrm{H}}(t)=\left(x_{i} \vee p_{i}\right)(t)$ and $\zeta_{i}^{\mathrm{I}}(t)=\left({\hat{x_{i}}}_{i}^{\mathrm{I}} \vee \hat{p}_{i}^{\mathrm{I}}\right)(t)$ are the three representations (SP, HP and IP) in RQM, while the dynamics of new systems $\frac{d \hat{\zeta}_{i}^{\mathrm{H}}(t)}{d t}$ is described from the following motion equations in NRNCQM:

$$
\begin{aligned}
\frac{d \zeta_{\mu}^{\mathrm{H}}(t)}{d t}=\left[\zeta_{\mu}^{\mathrm{H}}(t), \hat{H}_{\mathrm{rmp}}\right]+\frac{\partial \zeta_{\mu}^{\mathrm{H}}(t)}{\partial t} \\
\Longrightarrow \frac{d \hat{\zeta}_{\mu}^{\mathrm{H}}(t)}{d t}=\left[\hat{\zeta}_{\mu}^{\mathrm{H}}(t) * \hat{H}_{\mathrm{rnc}}^{\mathrm{mp}}\right]+\frac{\partial \hat{\zeta}_{\mu}^{\mathrm{H}}(t)}{\partial t} .
\end{aligned}
$$

The operators $\hat{H}_{\text {omp }}$ and $\hat{H}_{\text {rmp }}$ are the free and global Hamiltonians for the Morse potential model, while $\hat{H}_{\text {omp }}$ and $\hat{H}_{\mathrm{rnc}}^{\mathrm{mp}}$ are the corresponding Hamiltonians for the modified Morse potential. The present paper aims to extend the $l$-wave solutions by solving the modified Klein-Gordon equation with the modified Morse potential given in Refs. $[5,9,10,17]$ within the framework of Bopp's shift method and standard perturbation theory by taking an approximation to deal with the centrifugal potential term. The outline of the paper is organized as follows: Section II is mainly devoted to the basic eigenfunctions and the energy eigenvalues for the 
Morse potential in the relativistic Klein-Gordon equation. Section III is devoted to studying the modified Klein-Gordon equation MKGE by applying the ordinary Bopp's shift method and improved approximation of the centrifugal term to obtain the effective potential of the modified Morse potential. Besides, via perturbation theory, we find the expectation values of some radial terms to calculate the energy produced with the effect of the perturbed effective potential. Section IV is devoted to the global energy spectra produced with the modified Morse potential in the relativistic three-dimensional noncommutative real space (RNC: 3D-RS) symmetries. In Section $\mathrm{V}$, we treat the energy spectra for a few heterogeneous ( $\mathrm{LiH}, \mathrm{HCl}, \mathrm{NO}$ ) and homogeneous $\left(\mathrm{H}_{2}, \mathrm{I}_{2}, \mathrm{O}_{2}\right)$ diatomic molecules in a three-dimensional noncommutative nonrelativistic (NC: 3D-RS) symmetries under the modified Morse potential. Eventually, in Section VI, we presented our conclusion of this paper.

\section{REVISED EIGENFUNCTIONS AND ENERGY EIGENVALUES FOR THE MORSE POTENTIAL IN RQM}

The standard Morse potential given by Morse in Ref. [12], is useful to describing interatomic interaction of molecules and helpful in discussing polyatomic vibrational energies. An example is its application to the vibrational states of the heterogeneous ( $\mathrm{LiH}, \mathrm{HCl}, \mathrm{NO}$ ) and homogeneous $\left(\mathrm{H}_{2}, \mathrm{I}_{2}, \mathrm{O}_{2}\right)$ molecules. As already mentioned, our objective is to obtain the spectrum of the MKGE with the modified Morse potential in the relativistic three-dimensional noncommutative real space (RNC: 3D-RS) symmetries, we need to revise the corresponding Morse potential model in symmetries of ordinary relativistic quantum mechanics RQM:

$$
\left\{\begin{array}{l}
V_{\mathrm{mp}}(r)=a_{v}\left[1-\exp \left(-\alpha\left(r-r_{e}\right)\right)\right]^{2} \\
S_{\mathrm{mp}}(r)=a_{s}\left[1-\exp \left(-\alpha\left(r-r_{e}\right)\right)\right]^{2}
\end{array} .\right.
$$

To achieve this goal of our current research, it is useful to make a summary for the Klein-Gordon equation (KGE) with the Morse potential for a system of reduced mass $\mu=\frac{m_{1} m_{2}}{m_{1}+m_{2}}$ (for example, NO, $\mu=\frac{m_{\mathrm{N}} m_{\mathrm{O}}}{m_{\mathrm{N}}+m_{\mathrm{O}}}$ ) in three-dimensional relativistic quantum mechanics:

$$
\begin{gathered}
\left(\begin{array}{c}
-\Delta+\left(\mu+S_{\mathrm{mp}}(r)\right)^{2} \\
-\left(E_{n l}-V_{\mathrm{mp}}(r)\right)^{2}
\end{array}\right) \Psi(r, \theta, \varphi)=0 \\
\Longrightarrow \\
\left(\begin{array}{c}
\frac{d^{2}}{d r^{2}}+\frac{2}{r} \frac{d}{d r}+\left(E_{n l}^{2}-\mu^{2}\right) \\
-2\left(E_{n l} V_{\mathrm{mp}}(r)+\mu S_{\mathrm{mp}}(r)\right) \\
+V_{\mathrm{mp}}^{2}(r)-S_{\mathrm{mp}}^{2}(r)-\frac{l(l+1)}{r^{2}}
\end{array}\right) R_{n l}(r)=0 .
\end{gathered}
$$

The vector potential $V_{\mathrm{mp}}(r)$ is defined by the fourvector linear momentum operator $A^{\mu}\left(V_{\mathrm{mp}}(r), \mathbf{A}=\mathbf{0}\right)$ and the space-time scalar potential $S_{\mathrm{mp}}(r)$ is defined by the mass; $E_{n l}$ represent the relativistic energy eigenvalues in 3-dimensions and $l$ represents the principal and orbital quantum numbers, respectively. The Morse potential has spherical symmetry, allowing the solutions of the time-independent KGE of the known form $\Psi(r, \theta, \varphi)=R_{n l}(r) Y_{l}^{m}(\theta, \varphi)$ to separate the radial and angular parts of the wave function, and $\Delta$ is the ordinary 3-dimensional Laplacian operator. To eliminate the first order derivative, we introduce a new radial wave function $\chi_{n l}(r)=r R_{n l}(r)$ to the form, thus Eq. (2.2) becomes:

$$
\left(\begin{array}{c}
\frac{d^{2}}{d r^{2}}-\left(\mu^{2}-E_{n l}^{2}\right) \\
-2\left(\begin{array}{c}
E_{n l} V_{\mathrm{mp}}(r) \\
+\mu S_{\mathrm{mp}}(r)
\end{array}\right) \\
+V_{\mathrm{mp}}^{2}(r)-S_{\mathrm{mp}}^{2}(r)-\frac{l(l+1)}{r^{2}}
\end{array}\right) \chi_{n l}(r)=0 .
$$

The shorthand notation $E_{\mathrm{eff}}^{\mathrm{mp}}=\mu^{2}-E_{n l}^{2}$ and $V_{\mathrm{eff}}^{\mathrm{mp}}(r)=2\left(E_{n l} V_{\mathrm{mp}}(r)+\mu S_{\mathrm{mp}}(r)\right)-V_{\mathrm{mp}}^{2}(r)+$ $S_{\mathrm{mp}}^{2}(r)+\frac{l(l+1)}{r^{2}}$ we obtain the following second order Schrödinger-like equation:

$$
\left(\frac{d^{2}}{d r^{2}}-\left(E_{\mathrm{eff}}^{\mathrm{mp}}+V_{\mathrm{eff}}^{\mathrm{mp}}(r)\right)\right) \chi_{n l}(r)=0 .
$$

For the equal vector and scalar potential $V_{\mathrm{mp}}(r)=$ $S_{\mathrm{mp}}(r)$, the effective potential reduces to the form $V_{\mathrm{eff}}^{\mathrm{mp}}(r)=2\left(E_{n l}+\mu\right) V_{\mathrm{mp}}(r)+\frac{l(l+1)}{r^{2}}$. In the case of the nonrelativistic limit, Eq. (2.4) becomes a Schrödinger equation with the interaction potential $2 V_{\mathrm{mp}}(r)$. To aim for $V_{\mathrm{mp}}(r)$, not $2 V_{\mathrm{mp}}(r)$ in the interaction potential under the nonrelativistic limit, Xiang-Jun Xie et al. [10] apply the scheme proposed by Alhaidari et al. [39] to rescale the vector potential $V_{\mathrm{mp}}(r)$ and scalar potential $S_{\mathrm{mp}}(r)$, and rewrite Eq. (2.4) in the form of

$$
\left(\begin{array}{c}
\frac{d^{2}}{d r^{2}}-\left(\mu^{2}-E_{n l}^{2}\right) \\
+D_{e}\left[1-\exp \left(-\alpha\left(r-r_{e}\right)\right)\right]^{2}-\frac{l(l+1)}{r^{2}}
\end{array}\right) \chi_{n l}(r)=0 .
$$

As is mentioned in Ref. [10], Eq. (2.5) can be exactly solved when $l=0$. For the cases $l \neq 0$, there are only approximate solutions. Xiang-Jun Xie et al. applied the Pekeris approximation scheme given in Ref. [8, 40] to the centrifugal term as:

$$
\frac{l(l+1)}{r^{2}} \approx \frac{l(l+1)}{\mu r_{e}^{2}}\left(d_{0}+d_{1} \exp (-\alpha r)+d_{2} \exp (-2 \alpha r)\right),
$$

where the coefficients $d_{0}=1-\frac{3}{\alpha r_{e}}+\frac{3}{\alpha^{2} r_{e}^{2}}$, $d_{1}=\exp \left(2 \alpha r_{e}\right)\left(\frac{2}{\alpha r_{e}}-\frac{3}{\alpha^{2} r_{e}^{2}}\right)$ and $d_{2}=\exp \left(2 \alpha r_{e}\right)$ $\times\left(\frac{3}{\alpha^{2} r_{e}^{2}}-\frac{1}{\alpha r_{e}}\right)$ are determined from Refs. [9, 10, 17]. By substituting expression (2.6) into equation (2.5), XiangJun Xie et al. have obtained Eq. (12) in Refs. [9, 10]. So 
far, we are reforming this equation for a three-dimension space as follows:

$$
\left(\begin{array}{c}
d^{2} \\
d r^{2}
\end{array}+\left(\begin{array}{c}
\epsilon_{n l}-A_{n l} \exp (-\alpha r) \\
-B_{n l} \exp (-2 \alpha r)
\end{array}\right)\right) \chi_{n l}(r)=0
$$

where $A_{n l}=-2 D_{e}\left(E_{n l}+\mu\right) \exp \left(\alpha r_{e}\right)+\frac{l(l+1)}{\mu r_{e}^{2}} d_{1}$ and $B_{n l}=D_{e}\left(E_{n l}+\mu\right) \exp \left(2 \alpha r_{e}\right)+\frac{l(l+1)}{\mu r_{e}^{2}} d_{2}$ are given by [10]. Also, Ref. [10] gives the energy eigenvalues $E_{n l}$ of the KGE with equal scalar $S_{\mathrm{mp}}(r)$ and vector potential $V_{\mathrm{mp}}(r)$ and the corresponding total wave function as follows:

$$
\begin{aligned}
E_{n l}^{2}- & \mu^{2} \\
=- & {\left[\begin{array}{c}
\frac{2 D_{e}\left(E_{n l}+\mu\right) \exp \left(\alpha r_{e}\right)-\frac{l(l+1)}{\mu r_{e}^{2}} d_{1}}{2 \sqrt{\frac{l(l+1)}{\mu r_{e}^{2}} d_{2}+D_{e}\left(E_{n l}+\mu\right) \exp \left(2 \alpha r_{e}\right)}} \\
-(n+1 / 2) \alpha
\end{array}\right] } \\
& +D_{e}\left(E_{n l}+\mu\right)+\frac{l(l+1)}{\mu r_{e}^{2}} d_{0}
\end{aligned}
$$

and

$$
\begin{aligned}
\Psi(r, \theta, \varphi) & =\frac{\Gamma\left(1+b_{n l}\right) n l}{\Gamma\left(1+n+b_{n l}\right)} \frac{1}{r}\left(2 \beta_{n l} r\right)^{\frac{b_{n l}}{2}} \\
& \times \exp \left(-\beta_{n l} r\right) L_{n}^{b_{n l}}\left(2 \beta_{n l} r\right) Y_{l}^{m}(\theta, \varphi)
\end{aligned}
$$

where $b_{n l}=-2\left(n+1 / 2+\frac{A_{n l}}{2 \beta_{n l} \alpha^{2}}\right)=-\frac{\epsilon_{n l}}{\alpha^{2}}, \beta_{n l} \equiv \frac{\sqrt{B_{n l}}}{\alpha}$, $L_{n}^{b_{n l}}\left(2 \beta_{n l} r\right)$ are the associated Laguerre polynomials while $\epsilon_{n l}$ is given by:

$$
\epsilon_{n l}=E_{n l}^{2}-\mu^{2}-2 D_{e}\left(E_{n l}+\mu\right)-\frac{l(l+1)}{\mu r_{e}^{2}} d_{0} .
$$

\section{THE SOLUTION OF MKGE UNDER THE MODIFIED MORSE POTENTIAL IN (RNC: 3D-RS) SYMMETRIES}

At the beginning of this section, we shall define a formula of the modified Morse potential in the symmetries of a relativistic noncommutative three-dimensional real space (RNC: 3D-RS). To achieve this goal, it is useful to write the MKGE by applying the notion of WeylMoyal star product, which was shown previously in Eq. (1.3), to the differential equation that is satisfied by the radial wave function $\chi_{n l}(r)$ in Eq. (2.5), thus, the radial wave function $\chi_{n l}(r)$ in (RNC: $\left.3 \mathrm{D}-\mathrm{RS}\right)$ symmetries becomes as follows [41-48]:

$$
\begin{aligned}
& \left(\frac{d^{2}}{d r^{2}}+\left(\begin{array}{c}
\epsilon_{n l}-A_{n l} \exp (-\alpha r) \\
-B_{n l} \exp (-2 \alpha r)
\end{array}\right)\right) \chi_{n l}(r)=0 \\
& \Rightarrow \\
& \left(\frac{d^{2}}{d r^{2}}+\left(\begin{array}{c}
\epsilon_{n l}-A_{n l} \exp (-\alpha r) \\
-B_{n l} \exp (-2 \alpha r)
\end{array}\right)\right) * \chi_{n l}(r)=0 .
\end{aligned}
$$

It is well known that Bopp's shift method [48-53] has been applied effectively and has succeeded in simplifying the three basic equations: the modified Schrödinger equation (MSE), MKGE and the modified Dirac equation (MDE) with the notion of star product to the Schrödinger equation (SE), KGE and the Dirac equation (DE) with the notion of ordinary product, respectively. The application of this method was very useful and yielded promising results in many physical and chemical fields. The method reduced MSE, MKGE and MDE to the SE, KGE and DE, respectively, under the simultaneous translation in space-phase. The CNCCRs with star product in Eq. (1.2) become new CCCRs without the notion of star product as follows [43-50]:

$$
\begin{aligned}
{\left[\hat{x}_{\mu}^{\mathrm{S}}, \hat{x}_{\nu}^{\mathrm{S}}\right] } & =\left[\hat{x}_{\mu}^{\mathrm{H}}(t), \hat{x}_{\nu}^{\mathrm{H}}(t)\right]=\left[\hat{x}_{\mu}^{\mathrm{I}}(t), \hat{x}_{\nu}^{\mathrm{I}}(t)\right] \\
& =i \theta_{\mu \nu} .
\end{aligned}
$$

The generalized positions and momentum coordinates $\left(\hat{x}_{\mu}^{\mathrm{S}}, \hat{p}_{\mu}^{\mathrm{S}}\right),\left(\hat{x}_{\mu}^{\mathrm{H}}, \hat{p}_{i}^{\mathrm{H}}\right)(t)$ and $\left(\hat{x}_{\mu}^{\mathrm{I}}, \hat{p}_{\mu}^{\mathrm{I}}\right)(t)$ in the symmetries (RNC: 3D-RS) are defined in terms of the corresponding coordinates $\left(x_{\mu}^{\mathrm{S}}, p_{\mu}^{\mathrm{S}}\right),\left(x_{\mu}^{\mathrm{H}}, p_{i}^{\mathrm{H}}\right)(t)$ and $\left(x_{\mu}^{\mathrm{I}}, p_{\mu}^{\mathrm{I}}\right)(t)$ in RQM via, respectively (see, e.g., [52-58]):

$$
\left\{\begin{array}{l}
\left(x_{i}^{\mathrm{S}}, p_{i}^{\mathrm{S}}\right) \longrightarrow\left(\hat{x}_{\mu}^{\mathrm{S}}, \hat{p}_{\mu}^{\mathrm{S}}\right)=\left(x_{\mu}^{\mathrm{S}}-\frac{\theta^{\mu \nu}}{2} p_{\nu}^{\mathrm{S}}, p_{\mu}^{\mathrm{S}}\right) \\
\left(x_{i}^{\mathrm{H}}, p_{i}^{\mathrm{H}}\right) \longrightarrow\left(\hat{x}_{\mu}^{\mathrm{H}}, \hat{p}_{\mu}^{\mathrm{H}}\right)=\left(x_{\mu}^{\mathrm{H}}-\frac{\theta^{\mu \nu}}{2} p_{\nu}^{\mathrm{H}}, p_{\mu}^{\mathrm{H}}\right) \\
\left(x_{\mu}^{\mathrm{I}}, p_{\mu}^{\mathrm{I}}\right) \longrightarrow\left(\hat{x}_{\mu}^{\mathrm{I}}, \hat{p}_{\mu}^{\mathrm{I}}\right)=\left(x_{\mu}^{\mathrm{S}}-\frac{\theta^{\mu \nu}}{2} p_{\nu}^{\mathrm{I}}, p_{\mu}^{\mathrm{I}}\right)
\end{array}\right.
$$

This allows us to find the operator $r_{\mathrm{nc}}^{2}=r^{2}-\mathbf{L} \Theta$ in the symmetries of (RNC: 3D-RS) [56-59]. It is convenient to introduce a shorthand notation $r_{\mathrm{nc}} \rightarrow \hat{r}$, which will save us a lot of writing; the previous relation reduced to the $\hat{r}^{2}=r^{2}-\mathbf{L} \Theta$. The coupling $\mathbf{L} \Theta$ equals $\left(L_{x} \Theta_{12}+L_{y}\right.$ $\left.\Theta_{23}+L_{z} \Theta_{13}\right)$, here $\Theta_{i j}=\theta_{i j} / 2$ and $L_{x}, L_{y}$ and $L_{z}$ are the usual components of angular momentum operator in RQM. According to the Bopp shift method, Eq. (3.2) becomes similar to the following, like the Schrödinger equation (without the notions of star product):

$$
\begin{aligned}
& \left(\frac{d^{2}}{d r^{2}}+\left(\begin{array}{c}
\epsilon_{n l}-A_{n l} \exp (-\alpha r) \\
-B_{n l} \exp (-2 \alpha r)
\end{array}\right)\right) * \chi_{n l}(r)=0 \\
& \Rightarrow \\
& \left(\frac{d^{2}}{d r^{2}}+\left(\begin{array}{c}
\epsilon_{n l}-A_{n l} \exp (-\alpha \hat{r}) \\
-B_{n l} \exp (-2 \alpha \hat{r})
\end{array}\right)\right) \chi_{n l}(r)=0 .
\end{aligned}
$$

To simplify, we introduced a reduced-like Morse potential $V_{\mathrm{mp}}^{\mathrm{r}}(r)=A_{n l} \exp (-\alpha r)+B_{n l} \exp (-2 \alpha r)$. At the first order of the parameter $\Theta$, we apply the Taylor expansion as follows:

$$
V_{\mathrm{mp}}^{\mathrm{r}}(\hat{r})=V_{\mathrm{mp}}^{\mathrm{r}}(r)-\frac{\partial V_{\mathrm{mp}}^{\mathrm{r}}(r)}{\partial r} \frac{\mathbf{L} \Theta}{2 r}+O\left(\Theta^{2}\right) .
$$


Now, it is easy to obtain the following results:

$\frac{\partial V_{\mathrm{mp}}^{\mathrm{r}}(r)}{\partial r}=-\alpha A_{n l} \exp (-\alpha r)-2 \alpha B_{n l} \exp (-2 \alpha r)$.

This allows us to simplified the new differential in Eq. (3.4) to becomes as follows:

$$
\left(\begin{array}{c}
\frac{d^{2}}{d r^{2}}+\left(\begin{array}{c}
\epsilon_{n l}-A_{n l} \exp (-\alpha r) \\
-B_{n l} \exp (-2 \alpha r)
\end{array}\right) \\
-\alpha\left(A_{n l} \frac{\exp (-\alpha r)}{2 r}+B_{n l} \frac{\exp (-2 \alpha r)}{r}\right) \mathbf{L} \Theta
\end{array}\right) \chi_{n l}(r)=0 .
$$

Moreover, to illustrate the above equation in a simple mathematical way and an attractive form, it is useful to introduce the following symbol $V_{\mathrm{mp}}^{\mathrm{r}-\mathrm{nc}}(r)$, thus the radial Eq. (3.7) becomes:

$$
\left(\frac{d^{2}}{d r^{2}}+\left(\epsilon_{n l}-V_{\mathrm{mp}}^{\mathrm{r}-\mathrm{nc}}(r)\right)\right) \chi_{n l}(r)=0
$$

with:

$$
V_{\mathrm{mp}}^{\mathrm{r}-\mathrm{nc}}(r)=V_{\mathrm{mp}}^{\mathrm{r}}(r)+V_{\mathrm{pert}}^{\mathrm{rmp}}(r)
$$

Moreover, the second term is given by the following relation:

$$
V_{\text {pert }}^{\mathrm{rmp}}(r)=\alpha\left(A_{n l} \frac{\exp (-\alpha r)}{2 r}+B_{n l} \frac{\exp (-2 \alpha r)}{r}\right) \mathbf{L} \Theta .
$$

The reduced-like Morse potential is extended by including new terms proportional to the radial terms $\frac{\exp (-\alpha r)}{r}$ and $\frac{\exp (-2 \alpha r)}{r}$ to become a modified reducedlike ${ }^{r}$ Morse potential (MRLMP) in (RNC-3D: RSP) symmetries. The additive part $V_{\text {pert }}^{\text {rmp }}(r)$ of the new potential $V_{\mathrm{mp}}^{\mathrm{r}-\mathrm{nc}}(r)$ is proportional to the infinitesimal vector $\vec{\Theta}=\Theta_{12} e_{x}+\Theta_{23} e_{y}+\Theta_{13} e_{z}$. This allows us to consider the additive potential $V_{\text {pert }}^{\mathrm{rmp}}(r)$ as a perturbation potential compared with the main potential $V_{\mathrm{mp}}^{\mathrm{r}}(r)$ (parent potential operator) in the symmetries of (RNC: 3D-RS), that is, the inequality $V_{\text {pert }}^{\mathrm{rmp}}(r) \ll V_{\mathrm{mp}}^{\mathrm{r}}(r)$ has become achieved. In this way, all the physical justifications for applying the time-independent perturbation theory become satisfied. This allows us to give a complete prescription for determining the energy level of the generalized excited states. Now, we apply the perturbative theory in the case of RNCQM; we find the expectation values of the radial terms $\frac{\exp (-\alpha r)}{r}$ and $\frac{\exp (-2 \alpha r)}{r}$ taking into account the wave function which we have seen previously in Eq. (2.7). Thus, after straightforward calculations, we obtain the following results:

$$
\begin{aligned}
& \langle n, l, m\rangle \frac{\exp (-\alpha r)}{r}\langle n, l, m\rangle \equiv\left\langle\frac{\exp (-\alpha r)}{r}\right\rangle_{(n, l, m)} \\
& =2 \beta_{n l}\left[\frac{\Gamma\left(1+b_{n l}\right) n \downarrow}{\Gamma\left(1+n+b_{n l}\right)}\right]^{2} \int_{0}^{+\infty}\left(2 \beta_{n l} r\right)^{b_{n l}-1} \exp \left(-\left(\alpha+2 \beta_{n l}\right) r\right)\left[L_{n}^{b_{n l}}\left(2 \beta_{n l} r\right)\right]^{2} d r, \\
& \left\langle n, l, m\left|\frac{\exp (-2 \alpha r)}{r}\right| n, l, m\right\rangle \equiv\left\langle\frac{\exp (-2 \alpha r)}{r}\right\rangle_{(n, l, m)} \\
& =2 \beta_{n l}\left[\frac{\Gamma\left(1+b_{n l}\right) n \downarrow}{\Gamma\left(1+n+b_{n l}\right)}\right]^{2} \int_{0}^{+\infty}\left(2 \beta_{n l} r\right)^{b_{n l}-1} \exp \left(-2\left(\alpha+\beta_{n l}\right) r\right)\left[L_{n}^{b_{n l}}\left(2 \beta_{n l} r\right)\right]^{2} d r .
\end{aligned}
$$

We have applied the property of the spherical harmonics, which has the form $\int Y_{l}^{m}(\theta, \varphi) Y_{l^{\prime}}^{m^{\prime}}(\theta, \varphi) \sin \theta d \theta d \varphi=$ $\delta_{l l^{\prime}} \delta_{m m^{\prime}}$. For relieving the burden of writing, we will provide useful abbreviations $\langle n, l, m|A| n, l, m\rangle \equiv\langle A\rangle_{(n, l, m)}$. For the ground state $n=0$, we have $L_{0}^{b_{0 l}}\left(2 \beta_{n l} r\right)=1$, thus the above expectation values in Eq. (3.11) reduce to the following simple form:

$$
\left\{\begin{array}{l}
\left\langle\frac{\exp (-\alpha r)}{r}\right\rangle_{(0, l, m)}=\int_{0}^{+\infty} r^{\prime b_{0 l}-1} \exp \left(-\left(\frac{\alpha}{2 \beta_{0 l}}+1\right) r^{\prime}\right) d r^{\prime} \\
\left\langle\frac{\exp (-2 \alpha r)}{r}\right\rangle_{(0, l, m)}=\int_{0}^{+\infty} r^{\prime b_{0 l}-1} \exp \left(-\left(\frac{\alpha}{\beta_{0 l}}+1\right) r^{\prime}\right) d r^{\prime},
\end{array}\right.
$$


where $b_{0 l}=-2\left(1 / 2+\frac{A_{0 l}}{2 \beta_{0 l} \alpha^{2}}\right)=-\frac{\epsilon_{0 l}}{\alpha^{2}}, \beta_{0 l} \equiv \frac{\sqrt{B_{0 l}}}{\alpha}$, $A_{0 l}=-2 D_{e}\left(E_{0 l}+\mu\right) \exp \left(\alpha r_{e}\right)+\frac{l(l+1)}{\mu r_{e}^{2}} d_{1}, \quad B_{0 l}=$ $D_{e}\left(E_{0 l}+\mu\right) \exp \left(2 \alpha r_{e}\right)+\frac{l(l+1)}{\mu r_{e}^{2}} d_{2}$ while $\epsilon_{0 l}=E_{0 l}^{2}-\mu^{2}-$ $2 D_{e}\left(E_{0 l}+\mu\right)-\frac{l(l+1)}{\mu r_{e}^{2}} d_{0}$. Compare Eq. (3.12) with the integral of the form [60]:

$$
\int_{0}^{+\infty} x^{\nu-1} \exp \left(-\lambda x^{p}\right) d x=\frac{\lambda^{-\frac{\nu}{p}}}{p} \Gamma\left(\frac{\nu}{p}\right),
$$

where the conditions $\operatorname{Re}(\lambda)>0, \operatorname{Re}(\nu)>0$ and $p>0$ hold and $\Gamma\left(\frac{\nu}{p}\right)$ is the ordinary Gamma function. A direct calculation gives the expectation values in Eq. (3.19) as follows:

$$
\left\{\begin{array}{c}
\left\langle\frac{\exp (-\alpha r)}{r}\right\rangle_{(0, l, m)}=\left(\frac{\alpha}{2 \beta_{0 l}}+1\right)^{-b_{0 l}} \Gamma\left(b_{0 l}\right) \\
\left\langle\frac{\exp (-2 \alpha r)}{r}\right\rangle_{(0, l, m)}=\left(\frac{\alpha}{\beta_{0 l}}+1\right)^{-b_{0 l}} \Gamma\left(b_{0 l}\right)
\end{array} .\right.
$$

For the first excited state $n=1$, we have $L_{1}^{b_{1 l}}\left(2 \beta_{1 l} r\right)=$ $a+b r$, with $a=1+b_{1 l}, b=-2 \beta_{1 l}$, the expectation values in Eq. (3.11) are reduced to the following simple form:

$$
\begin{aligned}
& \left\langle\frac{\exp (-\alpha r)}{r}\right\rangle_{(1, l, m)}=\frac{2 \beta_{1 l}}{\left(1+b_{1 l}\right)^{2}} \int_{0}^{+\infty}\left(2 \beta_{1 l} r\right)^{b_{1 l}-1} \exp \left(-\left(\alpha+2 \beta_{1 l}\right) r\right)[a+b r]^{2} d r, \\
& \left\langle\frac{\exp (-2 \alpha r)}{r}\right\rangle_{(1, l, m)}=\frac{2 \beta_{1 l}}{\left(1+b_{1 l}\right)^{2}} \int_{0}^{+\infty}\left(2 \beta_{1 l} r\right)^{b_{1 l}-1} \exp \left(-2\left(\alpha+\beta_{1 l}\right) r\right)[a+b r]^{2} d r .
\end{aligned}
$$

We have used $\Gamma\left(2+b_{1 l}\right)=\left(1+b_{1 l}\right) \Gamma\left(1+b_{1 l}\right) . b_{1 l}=-2\left(3 / 2+\frac{A_{1 l}}{2 \beta_{1 l} \alpha^{2}}\right)=-\frac{\epsilon_{1 l}}{\alpha^{2}}, \beta_{1 l} \equiv \frac{\sqrt{B_{1 l}}}{\alpha}, A_{1 l}=$ $-2 D_{e}\left(E_{1 l}+\mu\right) \exp \left(\alpha r_{e}\right)+\frac{l(l+1)}{\mu r_{e}^{2}} d_{1}, B_{1 l}=D_{e}\left(E_{1 l}+\mu\right) \exp \left(2 \alpha r_{e}\right)+\frac{l(l+1)}{\mu r_{e}^{2}} d_{2}$ and $\epsilon_{1 l}=E_{1 l}^{2}-\mu^{2}-2 D_{e}\left(E_{1 l}+\mu\right)-$ $\frac{l(l+1)}{\mu r_{e}^{2}} d_{0}$. A direct simplification of Eq. (3.15) gives:

$$
\begin{gathered}
\left\langle\frac{\exp (-\alpha r)}{r}\right\rangle_{(1, l, m)}=\frac{2 \beta_{1 l}}{\left(1+b_{1 l}\right)^{2}}\left\{\begin{array}{c}
\frac{a^{2}}{2 \beta_{1 l}} \int_{0}^{+\infty} r^{\prime b_{1 l}-1} \exp \left(-\left(\frac{\alpha}{2 \beta_{1 l}}+1\right) r^{\prime}\right) d r^{\prime} \\
-\frac{a}{\beta_{1 l}} \int_{0}^{+\infty} r^{\prime b_{1 l}+1-1} \exp \left(-\left(\frac{\alpha}{2 \beta_{1 l}}+1\right) r^{\prime}\right) d r^{\prime} \\
+\frac{1}{2 \beta_{1 l}} \int_{0}^{+\infty} r^{\prime b_{1 l}+2-1} \exp \left(-\left(\frac{\alpha}{2 \beta_{1 l}}+1\right) r^{\prime}\right) d r^{\prime}
\end{array}\right\}, \\
\left\langle\frac{\exp (-2 \alpha r)}{r}\right\rangle_{(1, l, m)}=\frac{2 \beta_{1 l}}{\left(1+b_{1 l}\right)^{2}}\left\{\begin{array}{c}
\frac{a^{2}}{2 \beta_{1 l}} \int_{0}^{+\infty} r^{\prime b_{1 l}-1} \exp \left(-\left(\frac{\alpha}{\beta_{1 l}}+1\right) r^{\prime}\right) d r^{\prime} \\
-\frac{a}{\beta_{1 l}} \int_{0}^{+\infty} r^{\prime b_{1 l}+1-1} \exp \left(-\left(\frac{\alpha}{\beta_{1 l}}+1\right) r^{\prime}\right) d r^{\prime} \\
+\frac{1}{2 \beta_{1 l}} \int_{0}^{+\infty} r^{\prime b_{1 l}+2-1} \exp \left(-\left(\frac{\alpha}{\beta_{1 l}}+1\right) r^{\prime}\right) d r^{\prime}
\end{array}\right\} .
\end{gathered}
$$

We apply the integral in Eq. (3.13) to obtain the following results:

$$
\left\langle\frac{\exp (-\alpha r)}{r}\right\rangle_{(1, l, m)}=\left\{\begin{array}{c}
\left(\frac{\alpha}{2 \beta_{1 l}}+1\right)^{-b_{1 l}} \Gamma\left(b_{1 l}\right) \\
-\frac{2}{1+b_{1 l}}\left(\frac{\alpha}{2 \beta_{1 l}}+1\right)^{-\left(b_{1 l}+1\right)} \Gamma\left(b_{1 l}+1\right) \\
+\frac{2}{\left(1+b_{1 l}\right)^{2}}\left(\frac{\alpha}{2 \beta_{1 l}}+1\right)^{-\left(b_{1 l}+2\right)} \Gamma\left(b_{1 l}+2\right)
\end{array}\right\}
$$




$$
\left\langle\frac{\exp (-2 \alpha r)}{r}\right\rangle_{(1, l, m)}=\left\{\begin{array}{c}
\left(\frac{\alpha}{\beta_{1 l}}+1\right)^{-b_{1 l}} \Gamma\left(b_{1 l}\right) \\
-\frac{2}{1+b_{1 l}}\left(\frac{\alpha}{\beta_{1 l}}+1\right)^{-\left(b_{1 l}+1\right)} \Gamma\left(b_{1 l}+1\right) \\
+\frac{2}{\left(1+b_{1 l}\right)^{2}}\left(\frac{\alpha}{\beta_{1 l}}+1\right)^{-\left(b_{1 l}+2\right)} \Gamma\left(b_{1 l}+2\right)
\end{array}\right\} .
$$

The present study is divided into two main physical parts. The first part is to replace the coupling of the angular momentum operator with noncommutativity coupling $\mathbf{L} \Theta$ by the new interesting and significant equivalent coupling $(\Theta \vec{L} \vec{S} \equiv \Theta \mathbf{L S})$ with $\Theta=\left(\Theta_{12}^{2}+\Theta_{23}^{2}+\Theta_{13}^{2}\right)^{1 / 2}$ and the spinorbit coupling LS equals $L_{x} S_{x}+L_{y} S_{y}+L_{z} S_{z}$. Since $\vec{\Theta}$ is an arbitrary vector, we can choose it to be parallel to the spin $\vec{S}$ of the studied diatomic molecules. Moreover, we replace the new spinorbit coupling with a corresponding equivalent form $\frac{\Theta}{2}\left(\vec{J}^{2}-\vec{L}^{2}-\vec{S}^{2}\right)$. Thus the spin-orbit term contain- ing $V_{\text {pert }}^{\mathrm{r}-\text { so }}(r)=\alpha \Theta\left(A_{n l} \frac{\exp (-\alpha r)}{2 r}+B_{n l} \frac{\exp (-2 \alpha r)}{r}\right) \mathbf{L S}$. Furthermore, in the relativistic quantum mechanics (RQM), the operators $\left(H_{\mathrm{nc}-\mathrm{r}}^{\mathrm{mp}}, \vec{J}^{2}, \vec{L}^{2}, \vec{S}\right.$ and $\left.J_{z}\right)$ form a complete set of conserved physical quantities, the eigenvalues of the operator $\vec{J}^{2}-\vec{L}^{2}-\vec{S}^{2}$ are equal to the values $k(j, l, s)=j(j+1)-l(l+1)-$ $s(s+1)$. Consequently, the energies $\varepsilon_{0}^{\mathrm{mp}}(n=0, j, l, s)$ and $\varepsilon_{1}^{\mathrm{mp}}(n=1, j, l, s)$, due to the perturbed spin-orbit coupling $V_{\text {pert }}^{\mathrm{r}-\text { so }}(r)$ for the ground state and the first excited state, respectively, in (RNC: 3D-RS) symmetries are as follows:

$$
\left\{\begin{array}{c}
\varepsilon_{0}^{\mathrm{mp}}(n=0, j, l, s)=\alpha \Theta k(l)\left(\begin{array}{c}
\frac{A_{0 l}}{2}\left\langle\frac{\exp (-\alpha r)}{r}\right\rangle_{(0, l, m)} \\
\left.+B_{0 l} \frac{\exp (-2 \alpha r)}{r}\right\rangle_{(0, l, m)}
\end{array}\right) \\
\varepsilon_{1}^{\mathrm{mp}}(n=1, j, l, s)=\alpha \Theta k(l)\left(\begin{array}{c}
\frac{A_{1 l}}{2}\left\langle\frac{\exp (-\alpha r)}{r}\right\rangle_{(1, l, m)} \\
\left.+B_{1 l} \frac{\exp (-2 \alpha r)}{r}\right\rangle_{(1, l, m)}
\end{array}\right)
\end{array} .\right.
$$

This allows us to generalize the above results to the case of excited states of the diatomic $\left(\mathrm{H}_{2}, \mathrm{HCl}, \mathrm{I}_{2}, \mathrm{CO}, \mathrm{LiH}\right)$ and even polyatomic molecules under modified Morse potential in (RNC: 3D-RS) symmetries as follows:

$$
\varepsilon_{n}^{\mathrm{mp}}(n, j, l, s)=\alpha \Theta k(l)\left(\begin{array}{c}
\frac{A_{n l}}{2}\left\langle\frac{\exp (-\alpha r)}{r}\right\rangle_{(n, l, m)} \\
\left.+B_{n l} \frac{\exp (-2 \alpha r)}{r}\right\rangle_{(n, l, m)}
\end{array}\right) .
$$

The second part is to replace both $\left(\mathbf{L} \Theta\right.$ and $\left.\Theta_{12}\right)$ by $\left(\aleph \sigma L_{z}\right.$ and $\aleph \sigma$, respectively), we have chosen to orient the magnetic field $\aleph$ along the axis $(O z)$ to simplify the calculations, we also need to apply $\left\langle n^{\prime}, l^{\prime}, m^{\prime}\left|L_{z}\right| n, l, m\right\rangle=$ $\hbar m \delta_{n n^{\prime}} \delta_{l l^{\prime}} \delta_{m m^{\prime}}\left[\right.$ with $\left.-\left(l, l^{\prime}\right) \leqslant\left(m, m^{\prime}\right) \leqslant\left(l, l^{\prime}\right)\right]$. All of this data allows for the discovery of the new energy $\varepsilon_{0}^{\mathrm{mp}}(0, l, m)$ and $\varepsilon_{1}^{\mathrm{mp}}(1, l, m)$ due to the perturbed Zeeman effect generated by the influence of the perturbed effective potential $V_{\mathrm{nc} \text { eff }}^{\mathrm{mp}}(r)$ for the ground state and the first excited state in (RNC: 3D-RS) symmetries as follows:

$$
\left\{\begin{array}{c}
\varepsilon_{0}^{\mathrm{mp}}(n=0, l, m)=\alpha \aleph\left(\begin{array}{c}
\frac{A_{0 l}}{2}\left\langle\frac{\exp (-\alpha r)}{r}\right\rangle_{(0, l, m)} \\
\left.+B_{0 l} \frac{\exp (-2 \alpha r)}{r}\right\rangle_{(0, l, m)}
\end{array}\right) \sigma m \\
\varepsilon_{1}^{\mathrm{mp}}(n=1, l, m)=\alpha \aleph\left(\begin{array}{c}
\frac{A_{1 l}}{2}\left\langle\frac{\exp (-\alpha r)}{r}\right\rangle_{(1, l, m)} \\
\left.+B_{1 l} \frac{\exp (-2 \alpha r)}{r}\right\rangle_{(1, l, m)}
\end{array}\right) \sigma m
\end{array} .\right.
$$

Thus, we can generalize the previous results to the excited states of the diatomic $\left(\mathrm{H}_{2}, \mathrm{HCl}, \mathrm{I}_{2}, \mathrm{CO}, \mathrm{LiH}\right)$ and even polyatomic molecules under the modified Morse potential in (RNC: 3D-RS) symmetries as follows:

$$
\varepsilon_{n}^{\mathrm{mp}}(n, l, m)=\alpha \aleph\left(\begin{array}{c}
\frac{A_{n l}}{2}\left\langle\frac{\exp (-\alpha r)}{r}\right\rangle_{(n, l, m)} \\
\left.+B_{n l} \frac{\exp (-2 \alpha r)}{r}\right\rangle_{(n, l, m)}
\end{array}\right) \sigma m
$$




\section{THE GLOBAL RELATIVISTIC SPECTRUM OF THE MODIFIED MORSE POTENTIAL}

In this section, we report our results based on the superposition principle which permitted us to deduce the additive energy $\varepsilon_{0}^{\mathrm{r}-\mathrm{mp}}(n=0, j, l, m, s)$ and $\varepsilon_{1}^{\mathrm{r}-\mathrm{mp}}(n=1, j, l, m, s)$ for the ground state and the first excited state, respectively, in (RNC: 3D-RS) symmetries. Those energy shifts due to the spin-orbit coupling and the modified Zeeman effect are induced by $V_{\text {pert }}^{\text {rmp }}(r)$, the diatomic $\left(\mathrm{H}_{2}, \mathrm{HCl}, \mathrm{I}_{2}, \mathrm{CO}, \mathrm{LiH}\right)$ and even polyatomic molecules under the modified Morse potential as follows:

$$
\begin{aligned}
& \varepsilon_{0}^{\mathrm{r}-\mathrm{mp}}(n=0, j, l, m, s) \\
& =\alpha(\Theta k(l)+\aleph \sigma m)\left(\begin{array}{c}
\frac{A_{0 l}}{2}\left\langle\frac{\exp (-\alpha r)}{r}\right\rangle_{(0, l, m)} \\
\left.+B_{0 l} \frac{\exp (-2 \alpha r)}{r}\right\rangle_{(0, l, m)}
\end{array}\right), \\
& \varepsilon_{1}^{\mathrm{r}-\mathrm{mp}}(n=1, j, l, m, s) \\
& =\alpha(\Theta k(l)+\aleph \sigma m)\left(\begin{array}{c}
\frac{A_{1 l}}{2}\left\langle\frac{\exp (-\alpha r)}{r}\right\rangle_{(1, l, m)} \\
\left.+B_{1 l} \frac{\exp (-2 \alpha r)}{r}\right\rangle_{(1, l, m)}
\end{array}\right),
\end{aligned}
$$

which is easily generalized to the excited states in (RNC: 3D-RS) symmetries as follows:

$$
\begin{aligned}
& \varepsilon_{n}^{\mathrm{r}-\mathrm{mp}}(n, j, l, m, s) \\
& =\alpha(\Theta k(l)+\aleph \sigma m)\left(\begin{array}{c}
\frac{A_{n l}}{2}\left\langle\frac{\exp (-\alpha r)}{r}\right\rangle_{(n, l, m)} \\
\left.+B_{n l} \frac{\exp (-2 \alpha r)}{r}\right\rangle_{(n, l, m)}
\end{array}\right)
\end{aligned}
$$

The new modified radial part (new differential equation) of the MKGE in the symmetries of (RNC: 3D-RS) in Eq. (3.7) presents the physical system interacting with global potential $V_{\mathrm{nc}}^{\mathrm{r}-\mathrm{mp}}(r)$, which is composed of two parts, the first one is the main part $\left(V_{\mathrm{mp}}^{\mathrm{r}}(r)=\right.$ $A_{n l} \exp (-\alpha r)+B_{n l} \exp (-2 \alpha r)$ that is the reduced Morse potential in ordinary RQM) while the second part $V_{\text {pert }}^{\text {rmp }}(r)$ is in Eq. (3.10), this equivalent system has the relativistic energy in (RNC: 3D-RS):

$$
\begin{aligned}
\epsilon_{n l} & \rightarrow \varepsilon_{\mathrm{nc}}^{\mathrm{r}-\mathrm{mp}}\left(D_{e}, \alpha, r_{e}, n, j, l, m, s\right) \\
& =\epsilon_{n l}+\varepsilon_{n}^{\mathrm{r}-\mathrm{mp}}(n, j, l, m, s) .
\end{aligned}
$$

We replace $\epsilon_{n l}$ with the new values $\varepsilon_{\mathrm{nc}}^{\mathrm{r}-\mathrm{mp}}\left(D_{e}, \alpha, r_{e}, n, j, l, m, s\right)$ in Eq. (2.7) to obtain the modified relativistic energy as follows:

$$
\begin{aligned}
& \epsilon_{n l}+\varepsilon_{n}^{\mathrm{r}-\mathrm{mp}}(n, j, l, m, s) \\
= & E_{\mathrm{nc}}^{\mathrm{r}-\mathrm{mp} 2}-\mu^{2}-2 D_{e}\left(E_{n l}+\mu\right)-\frac{l(l+1)}{\mu r_{e}^{2}} d_{0} .
\end{aligned}
$$

Substituting expressions (4.2) and (4.3) into Eq. (4.4), we obtain the 3 -dimensional relativistic rotationvibrational energy equation for the diatomic $\left(\mathrm{H}_{2}, \mathrm{HCl}\right.$, $\mathrm{I}_{2}, \mathrm{CO}, \mathrm{LiH}$ ) and even polyatomic molecules in the presence of the equal scalar and vector modified Morse potentials in (RNC: 3D-RS) symmetries:

$$
\begin{aligned}
& E_{\mathrm{nc}}^{\mathrm{r}-\mathrm{mp} 2}-\mu^{2} \\
& =\epsilon_{n l}+\alpha(\Theta k(l)+\aleph \sigma m)\left(\begin{array}{c}
\frac{A_{n l}}{2}\left\langle\frac{\exp (-\alpha r)}{r}\right\rangle_{(n, l, m)} \\
\left.+B_{n l} \frac{\exp (-2 \alpha r)}{r}\right\rangle_{(n, l, m)}
\end{array}\right) \\
& +2 D_{e}\left(E_{\mathrm{nc}}^{\mathrm{r}-\mathrm{mp}}+\mu\right)+\frac{l(l+1)}{\mu r_{e}^{2}} d_{0} .
\end{aligned}
$$

\section{NONRELATIVISTIC TREATMENT OF THE MODIFIED MORSE POTENTIAL:}

\section{A. Nonrelativistic limit}

In order to consider further the interpretation of the positive and negative energy solutions of the modified Klein-Gordon equation, one can consider the nonrelativistic limit. For this purpose, we apply the following transformations:

$$
\left\{\begin{array}{l}
E_{\mathrm{nc}}^{\mathrm{r}-\mathrm{mp}}+\mu \rightarrow 2 \mu \\
E_{\mathrm{nc}}^{\mathrm{r}-\mathrm{mp}}-\mu \rightarrow E_{\mathrm{nc}}^{\mathrm{nr}-\mathrm{mp}}
\end{array} .\right.
$$

In order to apply the nonrelativistic limit, we reformulate the relativistic energy in (RNC: 3D-RS) symmetries (4.5) to the following form:

$$
\begin{aligned}
\left(E_{\mathrm{nc}}^{\mathrm{r}-\mathrm{mp}}+\mu\right)\left(E_{\mathrm{nc}}^{\mathrm{r}-\mathrm{mp}}-\mu\right) & =\epsilon_{n l}+\alpha(\Theta k(l)+\aleph \sigma m)\left(\begin{array}{c}
\frac{A_{n l}\left\langle\frac{\exp (-\alpha r)}{2}\right\rangle_{(n, l, m)}}{r} \\
\left.+B_{n l} \frac{\exp (-2 \alpha r)}{r}\right\rangle_{(n, l, m)}
\end{array}\right) \\
& +2 D_{e}\left(E_{\mathrm{nc}}^{\mathrm{r}-\mathrm{mp}}+\mu\right)+\frac{l(l+1)}{\mu r_{e}^{2}} d_{0}
\end{aligned}
$$

Substituting expression (5.1) into equation (5.2) yields the following equation:

$$
E_{\mathrm{nc}}^{\mathrm{nr}-\mathrm{mp}}=\frac{\epsilon_{n l}}{2 \mu}+\frac{\alpha}{2 \mu}(\Theta k(l)+\aleph \sigma m)\left(\begin{array}{c}
\frac{A_{n l}}{2}\left\langle\frac{\exp (-\alpha r)}{r}\right\rangle_{(n, l, m)} \\
\left.+B_{n l} \frac{\exp (-2 \alpha r)}{r}\right\rangle_{(n, l, m)}
\end{array}\right)+D_{e}+\frac{l(l+1)}{2 \mu^{2} r_{e}^{2}} d_{0} .
$$


Under nonrelativistic limit conditions, both $\left(A_{n l}\right.$ and $\left.B_{n l}\right)$ are reduced to $\left(-4 D_{e} \mu \exp \left(\alpha r_{e}\right)+\frac{l(l+1)}{\mu r_{e}^{2}} d_{1}\right.$ and $B_{n l}=$ $\left.D_{e} \mu \exp \left(2 \alpha r_{e}\right)+\frac{l(l+1)}{r_{e}^{2}} d_{2}\right)$, respectively, this allows us to reduce $\epsilon_{n l}$ to $\epsilon_{n l}^{\mathrm{nr}}$ the corresponding values as follows:

$$
\epsilon_{n l}=-2\left(n+1 / 2+\frac{A_{n l}}{2 \alpha \sqrt{B_{n l}}}\right) \rightarrow \epsilon_{n l}^{\mathrm{nr}}=-2\left(n+1 / 2-\frac{2 D_{e} \mu \exp \left(\alpha r_{e}\right)+\frac{l(l+1)}{\mu r_{e}^{2}} d_{1}}{\alpha \sqrt{D_{e} \mu \exp \left(2 \alpha r_{e}\right)+\frac{l(l+1)}{r_{e}^{2}} d_{2}}}\right)
$$

Substituting expression (5.4) into equation (5.3) yields the nonrelativistic expression of energy in (NC: 3D-RS) symmetries as follows:

$$
\begin{aligned}
E_{\mathrm{nc}}^{\mathrm{nr}-\mathrm{mp}} & =-\frac{1}{\mu}\left(\begin{array}{c}
n+1 / 2 \\
-\frac{2 D_{e} \mu \exp \left(\alpha r_{e}\right)+\frac{l(l+1)}{\mu r_{e}^{2}} d_{1}}{\alpha \sqrt{D_{e} \mu \exp \left(2 \alpha r_{e}\right)+\frac{l(l+1)}{r_{e}^{2}} d_{2}}}
\end{array}\right) \\
& +2 D_{e}+\frac{l(l+1)}{2 \mu^{2} r_{e}^{2}} d_{0}+\frac{\alpha}{2 \mu}(\Theta k(l)+\aleph \sigma m)\left(\begin{array}{c}
\frac{A_{n l}}{2}\left\langle\frac{\exp (-\alpha r)}{r}\right\rangle_{(n, l, m)} \\
\left.+B_{n l} \frac{\exp (-2 \alpha r)}{r}\right\rangle_{(n, l, m)}
\end{array}\right) .
\end{aligned}
$$

It should be noted that the first two terms in Eq. (5.5) are describing the nonrelativistic energy in the 3-dimensional relativistic rotation-vibrational energy equation for the diatomic $\left(\mathrm{H}_{2}, \mathrm{HCl}, \mathrm{I}_{2}, \mathrm{CO}, \mathrm{LiH}\right)$ and even polyatomic molecules in the presence of the equal scalar and vector Morse potentials in ordinary mechanics symmetries which confound with nonrelativistic energy under the standard Morse potential [5, 17].

\section{B. Nonrelativistic spectrum under the modified Morse potential}

Now, we want to derive the nonrelativistic spectrum, which is produced with the effect of the modified Morse potential for the diatomic molecules $\left(\mathrm{H}_{2}, \mathrm{HCl}, \mathrm{I}_{2}, \mathrm{CO}\right.$, $\mathrm{LiH})$ and even polyatomic molecules. From Eqs. (2.1) and (3.5), we can write the modified Morse potential in the nonrelativistic noncommutative three-dimensional real space (NC: 3D-RS) symmetries as follows:

$V_{\mathrm{nc}}^{\mathrm{mp}}(r)=V_{\mathrm{mp}}(r)+V_{\mathrm{mp}}^{\text {pert }}(r)$ with $V_{\mathrm{mp}}^{\text {pert }}(r) \ll V_{\mathrm{mp}}(r)$,

where the perturbative potential in (NC: 3D-RS) symmetries is:

$$
V_{\mathrm{mp}}^{\mathrm{pert}}(r)=-\frac{\partial V_{\mathrm{mp}}(r)}{\partial r} \frac{\mathbf{L} \Theta}{2 r}+O\left(\Theta^{2}\right)
$$

A direct calculation gives:

$$
\begin{aligned}
V_{\mathrm{mp}}^{\mathrm{pert}}(r) & =\frac{1}{2} D_{e} \alpha \exp \left(\alpha r_{e}\right) \\
& \times\left(\begin{array}{c}
\frac{\exp (-\alpha r)}{r} \\
-\exp \left(\alpha r_{e}\right) \frac{\exp (-2 \alpha r)}{r}
\end{array}\right) \mathbf{L} \Theta+O\left(\Theta^{2}\right)
\end{aligned}
$$

Thus, we need the expectation values of $\frac{\exp (-\alpha r)}{r}$ and $\frac{\exp (-2 \alpha r)}{r}$ to find the nonrelativistic energy corrections produced by the perturbative potential $V_{\mathrm{mp}}^{\text {pert }}(r)$. We have calculated the expectation values for the ground state and the first excited state and we generalized the results to the nth excited state. To avoid repetitions of the previous calculations and to respect the considerations about the corrections produced by the perturbed spin-orbit interaction and the modified Zeeman effect, we need to generalize the obtained energy in Eq. (4.2) to find the nonrelativistic global corrections $\Delta E_{\mathrm{nc}}^{\mathrm{nr}-\mathrm{mp}}(n, j, l, m, s)$ under the modified Morse potential for the diatomic $\left(\mathrm{H}_{2}, \mathrm{HCl}, \mathrm{I}_{2}, \mathrm{CO}, \mathrm{LiH}\right)$ and even polyatomic molecules as follows:

$$
\begin{aligned}
& \Delta E_{\mathrm{nc}}^{\mathrm{nr}-\mathrm{mp}}(n, j, l, m, s)=\alpha D_{e}(\Theta k(l)+\aleph \sigma m) \\
& \times\left(\begin{array}{c}
\exp \left(\alpha r_{e}\right)\left\langle\frac{\exp (-\alpha r)}{r}\right\rangle_{(n, l, m)} \\
-\exp \left(2 \alpha r_{e}\right)\left\langle\frac{\exp (-2 \alpha r)}{r}\right\rangle_{(n, l, m)}
\end{array}\right) .
\end{aligned}
$$

This allows us to find the nonrelativistic global energy $E_{\mathrm{nc}}^{\mathrm{nr}-\mathrm{mp}}\left(n, D_{e}, \alpha, r_{e}, j, l, m, s\right)$ under the modified Morse potential for the diatomic $\left(\mathrm{H}_{2}, \mathrm{HCl}, \mathrm{I}_{2}, \mathrm{CO}, \mathrm{LiH}\right)$ and even polyatomic molecules in the nonrelativistic noncommutative three-dimensional real space (NC: 3D$\mathrm{RS})$ symmetries as follows:

$$
\begin{aligned}
& E_{\mathrm{nc}}^{\mathrm{nr}-\mathrm{mp}}\left(n, D_{e}, \alpha, r_{e}, j, l, m, s\right) \\
& =E_{n l}^{\mathrm{nr}}+\alpha D_{e}(\Theta k(l)+\aleph \sigma m) \\
& \times\left(\begin{array}{c}
\exp \left(\alpha r_{e}\right)\left\langle\frac{\exp (-\alpha r)}{r}\right\rangle_{(n, l, m)} \\
-\exp \left(2 \alpha r_{e}\right)\left\langle\frac{\exp (-2 \alpha r)}{r}\right\rangle_{(n, l, m)}
\end{array}\right),
\end{aligned}
$$

where $E_{n l}^{\mathrm{nr}}$ is the nonrelativistic energy under the Morse potential for the diatomic $\left(\mathrm{H}_{2}, \mathrm{HCl}, \mathrm{I}_{2}, \mathrm{CO}, \mathrm{LiH}\right)$ and 
even polyatomic molecules. Now, considering composite systems such as molecules made of $N=2$ particles of masses $m_{n}(n=1,2)$ within the frame of noncommutative algebra, it is worth taking into account features of descriptions of the systems in the space. In a non-relativistic case, it was established that composite systems with different masses are described with different noncommutative parameters [61-63]:

$$
\begin{aligned}
& {\left[\hat{x}_{\mu}^{\mathrm{S}} * \wedge^{\mathrm{S}}{ }_{\nu}\right]=\left[\hat{x}_{\mu}^{\mathrm{H}}(t) * \wedge^{\mathrm{H}}(t)\right]=\left[\hat{x}_{\nu}^{\mathrm{I}}(t) * \wedge^{\mathrm{I}}(t)\right]} \\
& =i \theta_{\mu \nu}^{c},
\end{aligned}
$$

where the noncommutativity parameter $\theta_{\mu \nu}^{c}$ is given by:

$$
\theta_{\mu \nu}^{c}=\sum_{n=1}^{2} \mu_{n}^{2} \theta_{\mu \nu}^{(n)}
$$

with $\mu_{n}=\frac{m_{n}}{\sum_{n} m_{n}}$, the indices $(n=1,2)$ label the particle, and $\theta_{\mu \nu}^{(n)}$ is the parameter of noncommutativity, corresponding to the particle of mass $m_{n}$. Note that in the case of a system of two particles with the same mass $m_{1}=m_{2}$ such as the homogeneous $\left(\mathrm{H}_{2}, \mathrm{I}_{2}, \mathrm{O}_{2}\right)$ diatomic molecules, the parameter $\theta_{\mu \nu}^{(n)}=\theta_{\mu \nu}$. Thus, the two parameters $\Theta$ and $\sigma$, which appear in Eq. (5.10), are changed to the new form:

$$
\begin{aligned}
\Theta^{c 2}= & \left(\sum_{n=1}^{2} \mu_{n}^{2} \Theta_{12}^{(n)}\right)^{2}+\left(\sum_{n=1}^{2} \mu_{n}^{2} \Theta_{23}^{(n)}\right)^{2} \\
& +\left(\sum_{n=1}^{2} \mu_{n}^{2} \Theta_{13}^{(n)}\right)^{2}, \\
\sigma^{c 2}= & \left(\sum_{n=1}^{2} \mu_{n}^{2} \sigma_{12}^{(n)}\right)^{2}+\left(\sum_{n=1}^{2} \mu_{n}^{2} \sigma_{23}^{(n)}\right)^{2} \\
& +\left(\sum_{n=1}^{2} \mu_{n}^{2} \sigma_{13}^{(n)}\right)^{2}
\end{aligned}
$$

As is mentioned above, in the case of a system of two particles with the same mass $m_{1}=m_{2}$ such as the homogeneous $\left(\mathrm{H}_{2}, \mathrm{I}_{2}, \mathrm{O}_{2}\right)$ diatomic molecules, $\Theta_{\mu \nu}^{(n)}=\Theta_{\mu \nu}$ and $\sigma_{\mu \nu}^{(n)}=\sigma_{\mu \nu}$. Finally, we can generalize the nonrelativistic global energy $E_{\mathrm{nc}}^{\mathrm{nr}-\mathrm{mp}}\left(n, D_{e}, \alpha, r_{e}, j, l, m, s\right)$ under the modified Morse potential considering that composite systems with different masses are described with different noncommutative parameters for the diatomic $(\mathrm{HCl}, \mathrm{CO}$, $\mathrm{LiH})$ as:

$$
\begin{aligned}
& E_{\mathrm{nc}}^{\mathrm{nr}-\mathrm{mp}}\left(n, D_{e}, \alpha, r_{e}, j, l, m, s\right) \\
& =E_{n l}^{\mathrm{nr}}+\alpha D_{e}\left(\Theta^{c} k(l)+\aleph \sigma^{c} m\right) \\
& \times\left(\begin{array}{c}
\exp \left(\alpha r_{e}\right)\left\langle\frac{\exp (-\alpha r)}{r}\right\rangle_{(n, l, m)} \\
-\exp \left(2 \alpha r_{e}\right)\left\langle\frac{\exp (-2 \alpha r)}{r}\right\rangle_{(n, l, m)}
\end{array}\right) .
\end{aligned}
$$

It should be mentioned that for the two simultaneous limits $\left(\Theta^{c}, \sigma^{c}\right) \rightarrow(0,0)$, we recover the results of Refs. [8$10]$.

\section{CONCLUSIONS}

In this work, we have investigated the influence of the deformation of space due to the noncommutative quantum mechanics of space-time on the diatomic $\left(\mathrm{H}_{2}\right.$, $\mathrm{HCl}, \mathrm{I}_{2}, \mathrm{CO}, \mathrm{LiH}$ ) and even polyatomic molecules subject to a modified equal scalar and vector potential such as the Morse potential. The relativistic and nonrelativistic modified eigenvalues $E_{\mathrm{r}-\mathrm{nc}}^{\mathrm{mp}}\left(n, D_{e}, \alpha, r_{e}, j, l, m, s\right)$ and $E_{\mathrm{nc}}^{\mathrm{nr}-\mathrm{mp}}\left(n, D_{e}, \alpha, r_{e}, j, l, m, s\right)$ depend explicitly on the non-local parameter of the space-time $\left(\Theta^{c}, \sigma^{c}\right)$, in addition to the three parameters of the potential $\left(D_{e}, \alpha, r_{e}\right)$ and the discreet atomic quantum numbers. The presence of the new covariant noncommutative canonical commutations relations CNCCRs breaks the degeneracy of the spectrum of the standard Morse potential and replaces it with a new degeneracy of the spectrum. It is worth mentioning that, for all cases, when making the two limits $\left(\Theta^{c}, \sigma^{c}\right) \rightarrow(0,0)$ simultaneously, the ordinary physical quantities are recovered. The comparisons show that our theoretical results are in very good agreement with reported works.
[1] Z. H. Deng, Y. P. Fan, Shandong Univ. J. 7, 162 (1957).

[2] G. Pöschl, E. Teller, Z. Phys. 83, 143 (1933); https: //doi.org/10.1007/BF01331132.

[3] L. Z. Yi, Y. F. Diao, J. Y. Liu, C. S. Jia, Phys. Lett. A 333, 212 (2004); https://doi.org/10.1016/j.phys leta.2004.10.054.

[4] B. Fogarassy, G. Németh, Acta Phys. Acad. Sci. Hung. 11, 265 (1960); https://doi.org/10.1007/bf03158058.

[5] C. Berkdemir, J. Han, Chem. Phys. Lett. 409, 203 (2005); https://doi.org/10.1016/j.cplett.200
5.05 .021 .

[6] C. E. Burkhardt, J. J. Leventhal, Am. J. Phys. 75, 686 (2007); https://doi.org/10.1119/1.2750377.

[7] O. Bayrak, A. Soylu, I. Boztosun, J. Math. Phys. 51, 112301 (2010); https://doi.org/10.1063/1.3503413.

[8] S. M. Ikhdair, J. Math. Phys. 52, 052303 (2011); https: //doi.org/10.1063/1.3583553.

[9] Chun-Sheng Jia, Si-Yi Cao, Bull. Korean Chem. Soc. 34, 3425 (2013); https://doi.org/10.5012/bkcs.2013.34 .11 .3425 . 
[10] Xiang-Jun Xie, Chun-Sheng Jia, Phys. Scr. 90, 035207 (2015); https://doi.org/10.1088/0031-8949/90/3/0 35207.

[11] P. Zhang, H. C. Long, C. S. Jia, Eur. Phys. J. Plus 131, 117 (2016). https://doi.org/10.1140/epjp/i20 16-16117-4

[12] P. M. Morse, Phys. Rev. 34, 57 (1929); https://doi.or g/10.1103/PhysRev.34.57.

[13] S. Flügge, Practical Quantum Mechanics (Springer, Berlin, 1974).

[14] S. M. Ikhdair, R. Sever, Appl. Math. Comput. 218, 10082 (2012); https://doi.org/10.1016/j.amc. 2012 .03 .073 .

[15] S. H. Dong, in Factorization Method in Quantum Mechanics (Springer, Dordrecht), p. 73; https://doi. org /10.1007/978-1-4020-5796-0.

[16] P. O. Okoia, C. O. Edetb, T. O. Magu, Rev. Mex. Fís. 66, 1 (2020); https://doi .org/10.31349/RevMexFis .66.1.

[17] S. Miraboutalebi, L. Rajaei, J. Math. Chem. 52, 1119 (2014); https://doi.org/10.1007/s10910-014-03304.

[18] A. Adra, R. Server, Commun. Theor. Phys. 58, 27, (2012); https://doi.org/10.1088/0253-6102/58/1/0 5.

[19] S. Capozziello, G. Lambiase, G. Scarpetta, Int. J. Theor. Phys. 39, 15 (2000); https://doi.org/10.1023/A: 1003 634814685.

[20] S. Doplicher, K. Fredenhagen, J. E. Roberts, Phys. Lett. B 331, 39 (1994); https: //doi.org/10.1016/0370-269 3(94) 90940-7.

[21] E. Witten, Phys. Today 49, 24 (1996); https://doi.or g/10.1063/1.881493.

[22] A. Kempf, G. Mangano, R. B. Mann, Phys. Rev. D 52, 1108 (1995); https://doi.org/10.1103/physrevd.52. 1108.

[23] F. Scardigli, Nuovo Cim. B 110, 1029 (1995); https: //doi.org/10.1007/bf02726152.

[24] R. J. Adler, D. I. Santigo, Mod. Phys. Lett. A 14, 1371 (1999); https://doi.org/10.1142/s02177323990 01462.

[25] T. Kanazawa, G. Lambiase, G. Vilasi, A. Yoshioka, Eur. Phys. J. C 79, 95 (2019); https://doi.org/10.1140/ep jc/s10052-019-6610-1.

[26] F. Scardigli, Phys. Lett. B 452, 39 (1999); https://do i. org/10.1016/s0370-2693(99)00167-7.

[27] J. Zhang, Phys. Lett. B 584, 204 (2004); https://doi . org/10.1016/j.physletb.2004.01.049.

[28] J. Gamboa, M. Loewe, J. C. Rojas, Phys. Rev. D 64, 067901 (2001); https://doi.org/10.1103/PhysRevD.6 4.067901.

[29] M. Chaichian, Sheikh-Jabbari, A. Tureanu, Phys. Rev. Lett. 86, 2716 (2001); https://doi.org/10.1103/phys revlett.86.2716.

[30] M. A. De Andrade, C. Neves, J. Math. Phys. 59, 012105 (2018); https://doi.org/10.1063/1.4986964.

[31] J. Wang, K. Li, J. Phys. A 40, 2197 (2007); https://do i. org/10.1088/1751-8113/40/9/021.

[32] K. Li, J. Wang, Eur. Phys. J. C 50, 1007 (2007); https : //doi.org/10.1140/epjc/s10052-007-0256-0.

[33] H. Motavalli, A. R. Akbarieh, Mod. Phys. Lett. A 25, 2523 (2010); https://doi.org/10.1142/s02177323100 33529.

[34] A. Maireche, NanoWorld J. 1, 122 (2016); https://do i. org/10.17756/nwj . 2016-016.
[35] A. Maireche, J. Nano- Electron. Phys. 10, 06015 (2018).https://doi.org/10.21272/jnep. 10(6).06015.

[36] A. Maireche, J. Nano- Electron. Phys. 11, 04024 (2019); https://doi.org/10.21272/jnep.11(4).04024.

[37] J. Wang, K. Li, J. Phys. A 40, 2197 (2007); https://do i.org/10.1088/1751-8113/40/9/021.

[38] A. Saidi, M. B. Sedra, Mod. Phys. Lett. A 35, 2050014 (2020); https ://doi .org/10.1142/s021773232 0500145.

[39] A. D. Alhaidari, H. Bahlouli, A. Al-Hasan, Phys. Lett. A 349, 87 (2006); https://doi.org/10.1016/j.physle ta. 2005.09.008.

[40] C. L. Pekeris, Phys. Rev. 45, 98 (1934); https://doi. org/10.1103/physrev.45.98.

[41] A. Maireche, Afr. Rev. Phys. 15, 19 (2020).

[42] A. Maireche, Mod. Phys. Lett. A 35, 052050015 (2020); https://doi.org/10.1142/s0217732320500157.

[43] M. Darroodi, H. Mehraban, H. Hassanabadi, Mod. Phys. Lett. A 33, 1850203 (2018); https ://doi.org/10.1142/ s0217732318502036.

[44] A. Saidi, M. B. Sedra, Mod. Phys. Lett. A 35 , 2050014 (2020); https : //doi .org/10.1142/s021773232 0500145.

[45] A. Maireche, To Phys. J. 3, 186 (2019).

[46] A. Maireche, Afr. Rev. Phys. 15, 1 (2020).

[47] H. Motavalli, A. R. Akbarieh, Mod. Phys. Lett. A 25 2523 (2010); https://doi.org/10.1142/s02177323100 33529.

[48] L. Mezincescu, preprint arXiv: hep-th/0007046 (2000).

[49] E. F. Djemaï, H. Smail, Commun. Theor. Phys. 41, 837 (2004); https://doi.org/10.1088/0253-6102/41 $16 / 837$.

[50] J. Gamboa, M. Loewe, J. C. Rojas, Phys. Rev. D 64, 067901 (2001); https://doi.org/10.1103/PhysRevD.6 4.067901.

[51] E. M. C. Abreu, C. Neves, W. Oliveira, Int. J. Mod. Phys. A 21, 5359 (2006); https://doi.org/10.1142/s0 $217751 \times 06034094$.

[52] J. Zhang, Phys. Lett. B 584, 204 (2004); https://doi. org $/ 10.1016 / j$.physletb.2004.01.049.

[53] A. Maireche, J. Nano- Electron. Phys. 10, 06015 (2018).

[54] A. Maireche, To Phys. J. 5, 51 (2020); https://purkh. com/index.php/tophy/article/view/684.

[55] A. Maireche, Yanbu J. Engin. Sci. 16, 41 (2018).

[56] A. Maireche, Lat. Am. J. Phys. Educ. 9, 1301 (2015).

[57] A. Maireche, Int. Frontier Sci. Lett. 9, 33 (2016); https: //doi.org/10.18052/www.scipress.com/IFSL.9.33.

[58] A. Maireche, Sri Lankan J. Phys. 21, 11 (2020); https: //doi.org/10.4038/sljp.v21i1.8069.

[59] A. Maireche, Int. J. Geom. Meth. Mod. Phys. 17, 2050067 (2020); https : //doi .org/10.1142/S021988782 $050067 \mathrm{X}$.

[60] I. S. Gradshteyn, I. M. Ryzhik, Table of Integrals, Series and Products, 7th. ed. (University of Newcastle upon Tyne, England and Daniel Zwillinger, Rensselaer Polytechnic Institute USA, 2007).

[61] K. P. Gnatenko, V. M. Tkachuk, Europhys. Lett. 127, 20008 (2019). https://doi.org/10.1209/0295-5075/ $127 / 20008$.

[62] K. P. Gnatenko, V. M. Tkachuk, Int. J. Mod. Phys. A 33, 1850037 (2018); https://doi.org/10.1142/s02177 $51 \times 18500379$.

[63] K. P. Gnatenko, Phys. Lett. A 377, 3061 (2013); https: //doi.org/10.1016/j.physleta.2013.09.036. 
ЗВ'ЯЗАНІ СТАНИ МОДИФІКОВАНИХ РІВНЯНЬ КЛЯЙНА-ГОРДОНА ТА ШРЕДИНГЕРА ДЛЯ ДОВІЛЬНОГО L-СТАНУ 3 МОДІФІКОВАНИМ ПОТЕНЦІАЛОМ МОРЗЕ В СИМЕТРІЯХ НЕКОМУТАТИВНОї КВАНТОВОї МЕХАНІКИ

\begin{abstract}
Абдельмаджід Майреш
Лабораторіл фізики та хімї̈ матеріалів, фізичне відділеннл,

Факулътет природничих наук, Університет Мсіли, Мсіла, Алжир (ВР 239 Шебілія-Мсіла, Алжир)

У цій роботі отримано наближені аналітичні розв'язки як модифікованого рівняння КляйнаГордона, так і рівняння Шредингера в некомутативному релятивістському та нерелятивістському тривимірному дійсному просторі. 3 цією метою використано схему наближення Пекеріса для врахування відцентрового доданка, метод зсуву Боппа та стандартну теорію збурень. Ми отримуємо рівняння на енергію зв'язаного стану з нещодавно запропонованим потенціалом, який називається модифікованим потенціалом Морзе, за умови однакових скалярних та векторних потенціалів. Цей потенціал є суперпозицією потенціалу Морзе та деяких експоненційних радіальних доданків. Метою поєднання цих потенціалів $є$ широке застосування. Ми показуємо, що нова енергія залежить від глобальних параметрів $\left(\Theta^{c}\right.$ та $\left.\sigma^{c}\right)$, які характеризують некомутативний простір-простір, i параметрів потенціалу $\left(D_{e}, r_{e}, \alpha\right)$ на додаток до гамма-функції та дискретних атомних квантових чисел $(j, l, s, m)$. Наведені результати застосовують для розрахунку енергетичного спектра кількох неоднорідних ( $\mathrm{LiH}, \mathrm{HCl}, \mathrm{NO})$ та однорідних $\left(\mathrm{H}_{2}, \mathrm{I}_{2}, \mathrm{O}_{2}\right)$ двохатомних молекул. Ми також обговорюємо деякі фізично важливі випадки.
\end{abstract}

Ключові слова: рівняння Кляйна-Гордона, рівняння Шредингера, потенціал Морзе, некомутативна квантова механіка, зіркові добутки. 